\title{
Eficácia comparativa de duas formulaçóes de uso tópico contendo fipronil $10 \%$ no controle de Ctenocephalides felis felis em gatos
}

\author{
Comparative efficacy of two topical formulations containing $10 \%$ fipronil \\ on the control of Ctenocephalides felis felis on cats. \\ Michelle Godan de Freitas Tancredi ${ }^{1}$; Thaís Ribeiro Correia ${ }^{2}$; Francisco de Assis Ribeiro; \\ Maria Clara da Silva Negreiros Botelho ${ }^{4}$; Pedro Vianna Tavares ${ }^{3}$; Fabio Barbour Scott ${ }^{5 *}$; \\ Guilherme Gomes Verocai ${ }^{6}$; Katherina Coumendouros ${ }^{5}$
}

\author{
${ }^{1}$ Faculdade de Medicina Veterinária de Valença, Fundaçăo Educacional Dom André Arcoverde, Centro de Ensino Superior de Valença \\ ${ }^{2}$ Programa de Pós-doutoramento, Departamento de Parasitologia Animal, Instituto de Veterinária, \\ Universidade Federal Rural do Rio de Janeiro - UFRRJ \\ ${ }^{3}$ Curso de Pós-Graduação em Ciências Veterinárias, Departamento de Parasitologia Animal, Instituto de Veterinária, \\ Universidade Federal Rural do Rio de Janeiro - UFRRJ \\ ${ }^{4}$ Curso de Pós-Graduação em Medicina Veterinária, Departamento de Medicina e Cirurgia Veterinária, Instituto de Veterinária, \\ Universidade Federal Rural do Rio de Janeiro - UFRRJ \\ ${ }_{5}^{5}$ Departamento de Parasitologia Animal, Instituto de Veterinária, Universidade Federal Rural do Rio de Janeiro - UFRRJ \\ ${ }^{6}$ Médico Veterinário MSc
}

Recebido em 3 de Fevereiro de 2009

Aceito em 16 de Abril de 2009

\section{Resumo}

No presente trabalho, foram avaliadas, comparativamente, duas formulaçóes comerciais tópicas, contendo $10 \%$ de fipronil, para o controle da pulga Ctenocephalides felis felis em gatos. O experimento foi realizado no Laboratório de Quimioterapia Experimental em Parasitologia Veterinária do Departamento de Parasitologia Animal, Instituto de Veterinária, da Universidade Federal Rural do Rio de Janeiro. Foram empregados 18 gatos, sem raça definida, divididos em três grupos de seis. Um grupo permaneceu sem tratamento (controle), e os demais foram medicados com uma formulaçáo referência do mercado veterinário ou com uma nova formulaçáo em teste. Os animais tratados receberam $0,5 \mathrm{~mL}$ do produto ao longo da região dorso-cervical e foram infestados nos dias $-2,+5,+12,+19,+26$ e +33 . A primeira avaliação foi realizada quatro dias após a primeira infestação e, as demais, 48 horas após cada infestação, por meio do comb test. Ambas as formulaçóes testadas foram eficazes até o dia +35 após o tratamento, não havendo diferenças entre seus níveis de eficácia ao longo do período experimental. A nova formulação, contendo fipronil a 10\%, apresentou a eficácia desejada no controle de $C$. $f$. felis em gatos.

Palavras-chave: Controle, fipronil, Ctenocephalides felis felis.

\begin{abstract}
The goal of the present study was to evaluate the comparative efficacy of two topical formulations containing $10 \%$ fipronil on the control of Ctenocephalides felis felis on cats. The trial was performed at the Laboratory of Experimental Chemotherapy in Veterinary Parasitology from the Department of Animal Parasitology of the Institute of Veterinary of the Universidade Federal Rural do Rio de Janeiro. Eighteen mixed-breed cats were divided in three groups of six animals each. One group remained without treatment (control). The other groups received as treatment the two topical formulations, a commercial reference and the novel one, both containing $10 \%$ fipronil. Treated animals received $0.5 \mathrm{~mL}$ of product along their cervical region. Cats were infested on days $-2,+5,+12,+19,+26$ and +33 . Evaluations were made using the "comb test". The first one was undertaken 4 days after the initial infestation $($ day +2$)$, and others 48 hours after following infestations (days $+7,+14,+21,+28$ and +35 ). Both tested formulations had satisfactory efficacy until day +35 . No differences were observed comparing the efficacy levels between both formulations troughout the experimental period. The novel topical $10 \%$ fipronil formulation presented desirable efficacy on the control of $C$. $f$. felis on cats.
\end{abstract}

Keywords: Control, fipronil, Ctenocephalides felis felis.

\footnotetext{
*Autor para correspondência: Fabio Barbour Scott

Professor Adjunto, Departamento de Parasitologia Animal - DPA,

Instituto de Veterinária, Universidade Federal Rural do Rio de Janeiro - UFRRJ,

CEP 23890-000, Rio de Janeiro - RJ, Brasil

e-mail:scott@ufrrj.br
} 
Devido a sua relevante importância médico-veterinária e crescente papel em Saúde Pública, a pulga Ctenocephalides felis felis tem sido alvo de numerosos estudos relacionados à biologia, controle, transmissão de patógenos aos animais domésticos e ao homem. No que tange ao controle, grande enfoque vem sendo dado ao estudo dos fatores que interferem no ciclo biológico e estudos relacionados à ação dos compostos químicos, como pulicidas (DRYDEN; PRESTWOOD, 1993; RUST, 2005; OTRANTO; WALL, 2008).

Os fenilpirazóis foram desenvolvidos na década de 80 e introduzidos no mercado nos anos 90 com diversas indicaçóes, tanto para uso agrícola como para a medicina veterinária (TANNER et al., 1997; CHANDLER et al., 2004). A principal molécula empregada dessa classe no controle ectoparasitário em animais domésticos é o fipronil (SCOTT et al., 2002), eficaz no controle de pulgas em gatos (HUTCHINSON et al., 1998). Mais recentemente, um novo composto desse grupamento, o piriprole, também passou a ser utilizado no controle de ectoparasitos de cáes e gatos (BARNETT et al., 2008; SCHUELE et al., 2008).

O objetivo do presente trabalho foi avaliar comparativamente duas formulações, uma de referência e outra de aplicação tópica, contendo $10 \%$ de fipronil, no controle da pulga $C$. $f$. felis em gatos.

$\mathrm{O}$ experimento foi realizado nas dependências do Laboratório de Quimioterapia Experimental em Parasitologia Veterinária (LQEPV), do Departamento de Parasitologia Animal, Instituto de Veterinária, da Universidade Federal Rural do Rio de Janeiro.

Foram empregados 18 gatos, adultos, sem raça definida, machos e fêmeas, com peso corporal variando de dois a quatro quilos. Os animais foram alojados em gaiolas individuais suspensas sete dias antes do início dos ensaios (dia -7). Nessa data, foram infestados com 50 pulgas ( $C . f$. felis) adultas não alimentadas, com 14 dias de idade, oriundas da colônia mantida no LQEPV. Após 48 horas $\mathrm{da}$ infestação, todos os animais foram penteados (comb test), e as pulgas retiradas foram quantificadas. Baseada na contagem preliminar, foi elaborada a randomização. Efetuou-se um sorteio de cada animal, do mais parasitado para o menos parasitado, até completar os três grupos. Posteriormente, foi realizado o sorteio dos grupos referente aos tratamentos, assim constituídos:

- Um grupo medicado com a formulação referência, contendo 10\% de fipronil, (Frontline ${ }^{\circledR}$ TopSpot - Merial Saúde Animal), empregando-se um volume total de $0,5 \mathrm{~mL}$ do produto, aplicados diretamente na pele do animal, ao longo da regiấo dorso-cervical, conforme recomendação do fabricante;

- Um grupo medicado com uma formulação em teste, contendo $10 \%$ de fipronil (Fiprolex ${ }^{\circledR}$ Drop Spot - Sespo Indústria e Comércio Ltda.), empregando-se um volume total de $0,5 \mathrm{~mL}$ do produto, aplicados diretamente na pele do animal, ao longo da região dorso-cervical, conforme recomendação do fabricante;

- Um grupo foi mantido sem tratamento, o grupo controle. Em seguida, no dia -7, cada gato foi infestado com 100 pulgas adultas não-alimentadas oriundas da colônia. As infestaçôes ocorreram nos dias $-2,+5,+12,+19,+26$ e +33 . Os animais foram avaliados nos dias $+2,+7,+14,+21,+28$ e +35 , com o auxílio de um pente fino próprio para retirada de pulgas. As pulgas recuperadas foram quantificadas e fixadas em álcool $70{ }^{\circ} \mathrm{GL}$.
A eficácia pulicida foi calculada com base na Equação 1:

$\begin{gathered}\text { Percentagem } \\ \text { de eficácia }\end{gathered}=\left(\begin{array}{l}\text { m.a. de pulgas vivas recuperadas no g.c. }- \\ \text { m.a. de pulgas vivas recuperadas no g.m. } \\ \text { m.a. de pulgas vivas recuperadas no g.c. }\end{array}\right) \times 100$

m.a. - média aritmética; g.c. - grupo controle; g.m. - grupo medicado

A metodologia empregada no estudo foi a preconizada pela Associação Mundial para Avanço da Parasitologia Veterinária (MARCHIONDO et al., 2007)

Os resultados obtidos nas avaliaçóes foram transformados em $\log (x+1)$ e submetidos à análise de variância seguido do Teste de Tukey, quando as variâncias fossem desiguais. O nível de significância considerado foi de 95\% (P < 0,05) (SAMPAIO, 2002). As análises estatísticas foram realizadas pelos programas estatísticos computacionais BioEstat 4.0 (AYRES et al., 2005).

As contagens de pulgas, antes e após o tratamento, assim como as médias, desvio padrão e eficácia estão contidos na Tabela 1 .

As médias do grupo controle foram 32,$3 ; 19,2 ; 22,5 ; 30,7 ; 30,7$ e 30,3 para os dias $+2,+7,+14,+21,+28$ e +35 , respectivamente.

As médias do grupo tratado com a formulação referência de fipronil foram 0,5; zero; zero; 0,$3 ; 3,2$ e 7,7 para os dias $+2,+7,+14$, $+21,+28$ e +35 , respectivamente. Foi patente a queda do número de pulgas deste grupo, pelos baixos níveis de infestaçóes apresentados até o dia +35 . Comparativamente, as médias do controle e da formulação referência de fipronil diferiram estatisticamente para todos os dias de avaliaçóes $(\mathrm{P} \leq 0,05)$. As eficácias do tratamento foram 98,$4 ; 100 ; 100 ; 99,2 ; 89,6 ; 74,6 \%$ para os dias $+2,+7$, $+14,+21,+28$ e +35 , respectivamente.

As médias do grupo tratado com a formulação teste de fipronil foram 0,2 ; zero; zero; zero; 1,$5 ; 4,0$; respectivamente para os dias $+2,+7,+14,+21,+28$ e +35 , demonstrando assim baixos níveis de infestaçóes. Comparativamente ao grupo controle, as médias do grupo tratado com a formulação nova em teste de fipronil diferiram estatisticamente para todos os dias de avaliaçóes $(\mathrm{P} \leq 0,05)$. As eficácias do tratamento foram de $99,4 \%$ para o dia $+2 ; 100 \%$ para os dias $+7,+14$ e $+21 ; 95,1 \%$ para o dia +28 e $86,8 \%$ para o dia +35 .

Comparando-se estatisticamente as médias de pulgas vivas do grupo tratado com a formulação teste e a formulação referência de fipronil não houve diferença $(\mathrm{P}>0,05)$ durante todo o período experimental, caracterizando proteção contra reinfestaçóes para ambas.

Medleau et al. (2002) empregaram três tratamentos com intervalos mensais em gatos e obtiveram eficácia de 75, 73, 85 e $94 \%$ nos dias $+14,+30,+60$ e +90 , respectivamente. No entanto, podem ser confrontados os resultados obtidos para o dia +14 , pois até tais períodos observacionais, as metodologias empregadas foram equivalentes. Portanto, pode-se afirmar que, tanto para formulação teste de fipronil quanto para a de referência, as eficácias obtidas neste estudo foram superiores às obtidas pelo estudo de Medleau et al. (2002). Para a comparação da eficácia obtida por Medleau et al. (2002) no dia +30 , pode-se confrontá-la com os índices de eficácia alcançados nos dias +28 e +35 do presente estudo, $95,1 \%$ e $86,6 \%$, respectivamente, para formulação teste, e $89,6 \%$ e $74,6 \%$ para a de referência, que ainda se apresentaram superiores. 
Tabela 1. Eficácia comparativa entre as formulaçóes teste e referência à base de fipronil $10 \%$ no controle da pulga Ctenocephalides felis felis em gatos.

\begin{tabular}{|c|c|c|c|c|c|c|}
\hline \multirow{2}{*}{ Grupo/Animal } & \multicolumn{6}{|c|}{ Número de pulgas recuperadas por dia de desafio } \\
\hline & Dia +2 & Dia +7 & Dia +14 & Dia +21 & Dia +28 & Dia +35 \\
\hline \multicolumn{7}{|l|}{ Controle } \\
\hline $1 \mathrm{C}$ & 29 & 13 & 7 & 10 & 17 & 20 \\
\hline $2 \mathrm{C}$ & 57 & 29 & 21 & 41 & 25 & 61 \\
\hline $3 \mathrm{C}$ & 28 & 24 & 31 & 36 & 39 & 30 \\
\hline $4 \mathrm{C}$ & 41 & 30 & 30 & 37 & 27 & 22 \\
\hline $5 \mathrm{C}$ & 18 & 5 & 21 & 32 & 41 & 22 \\
\hline $6 \mathrm{C}$ & 21 & 18 & 25 & 28 & 35 & 27 \\
\hline Média $\pm \mathrm{DP}^{1}$ & $32,3^{a} \pm 13,2$ & $19,2^{\mathrm{a}} \pm 8,9$ & $22,5^{\mathrm{a}} \pm 8,0$ & $30,7^{a} \pm 10,1$ & $30,7^{\mathrm{a}} \pm 8,4$ & $30,3^{\mathrm{a}} \pm 14,1$ \\
\hline \multicolumn{7}{|l|}{$\begin{array}{l}\text { Formulação } \\
\text { referência }\end{array}$} \\
\hline $1 \mathrm{~B}$ & 0 & 0 & 0 & 0 & 0 & 6 \\
\hline $2 \mathrm{~B}$ & 2 & 0 & 0 & 0 & 0 & 2 \\
\hline $3 \mathrm{~B}$ & 0 & 0 & 0 & 0 & 16 & 22 \\
\hline $4 \mathrm{~B}$ & 1 & 0 & 0 & 0 & 0 & 3 \\
\hline $5 \mathrm{~B}$ & 0 & 0 & 0 & 0 & 1 & 6 \\
\hline $6 \mathrm{~B}$ & 0 & 0 & 0 & 2 & 2 & 7 \\
\hline Média \pm DP & $0,5^{\mathrm{b}} \pm 0,8$ & $0^{\mathrm{b}} \pm 0$ & $0^{\mathrm{b}} \pm 0$ & $0,3^{\mathrm{b}} \pm 0,7$ & $3,2^{\mathrm{b}} \pm 5,8$ & $7,7^{\mathrm{b}} \pm 6,6$ \\
\hline Eficácia (\%) & 98,4 & 100 & 100 & 99,2 & 89,6 & 74,6 \\
\hline \multicolumn{7}{|l|}{ Formulação teste ${ }^{3}$} \\
\hline $1 \mathrm{~A}$ & 1 & 0 & 0 & 0 & 6 & 6 \\
\hline $2 \mathrm{~A}$ & 0 & 0 & 0 & 0 & 0 & 0 \\
\hline $3 \mathrm{~A}$ & 0 & 0 & 0 & 0 & 0 & 3 \\
\hline $4 \mathrm{~A}$ & 0 & 0 & 0 & 0 & 0 & 4 \\
\hline $5 \mathrm{~A}$ & 0 & 0 & 0 & 0 & 0 & 2 \\
\hline $6 \mathrm{~A}$ & 0 & 0 & 0 & 0 & 3 & 9 \\
\hline Média \pm DP & $0,2^{\mathrm{b}} \pm 0,4$ & $0^{\mathrm{b}} \pm 0$ & $0^{\mathrm{b}} \pm 0$ & $0^{\mathrm{b}} \pm 0$ & $1,5^{\mathrm{b}} \pm 2,3$ & $4,0^{\mathrm{b}} \pm 2,9$ \\
\hline Eficácia (\%) & 99,4 & 100 & 100 & 100 & 95,1 & 86,8 \\
\hline
\end{tabular}

${ }^{1}$ Desvio padrão; ${ }^{2}$ Frontline ${ }^{\circledR}$ TopSpot (Merial Saúde Animal); ${ }^{3}$ Fiprolex ${ }^{\circledR}$ Drop Spot (Sespo Indústria e Comércio Ltda); Médias entre colunas com letras iguais não diferem significativamente $(\mathrm{P}>0,05)$

Alguns estudos avaliam principalmente o efeito de knock down sobre o inseto exercido pelo inseticida. Schenker et al. (2003) observaram que o fipronil top spot $10 \%$ apresentou eficácia de 24,3 e $62,2 \%$ nos intervalos de 3 e 8 horas após o tratamento em gatos

Franc e Yao (2007) avaliaram a eficácia adulticida da mesma formulação referência de fipronil no controle de $C$. $f$. felis e C. f. strongylus em gatos, comparativamente com as eficácias da selamectina e do imidacloprid. Esses autores empregaram uma metodologia bastante semelhante ao do presente estudo, sendo apenas diferente quanto aos dias de reinfestaçóes e avaliaçóes. $\mathrm{O}$ fipronil obteve eficácia de $100 \%$ para ambas as subespécies entre os dias +9 e +37 , corroborando, em parte, os resultados do presente estudo, pois apresentou mais elevados níveis de eficácia em torno da quinta e sexta semanas após tratamento.

Payne et al. (2001) avaliaram uma formulação spray contendo fipronil 0,29\% em pulgas de três diferentes linhagens laboratoriais. Obtiveram eficácias superiores a 98,2\% para todas as linhagens até o dia +16 . No dia +23 , duas linhagens continuaram com eficácia acima de $98,4 \%$ e outra $88,8 \%$, diferindo significativamente das demais, porém ainda sendo considerada elevada. No último dia de avaliação, dia +30 , a eficácia do fipronil diferiu significativamente entre todas as linhagens, porém permaneceram superiores a $77,3 \%$. Além de empregarem uma formulação spray, e de os dias de infestação e avaliação não coincidirem com os do presente ensaio, ambos com dois dias de atraso, os índices de eficácia se mostraram satisfatórios e similares até a quarta e quinta semanas após tratamento. Adicionalmente, tal trabalho mostra que diferentes linhagens de pulga podem apresentar diferentes susceptibilidades ao fipronil, o que reforça a necessidade de realização de testes com as mesmas formulaçóes em diversos países ou regióes de um mesmo país ou mesmo diferentes linhagens laboratoriais, principalmente quando são testes visando ao registro de novas formulaçóes voltadas ao controle de ectoparasitos para o mercado médico-veterinário. 
As duas formulações de fipronil não apresentaram diferença nos níveis de eficácia observados e foram eficazes no controle da pulga $C$. felis felis em gatos por até 35 dias após o tratamento.

\section{Referências}

AYRES, M. et al. BioEstat 4.0 - Aplicaçóes estatísticas nas áreas das ciências biológicas e médicas. 4 ed. Belém: Sociedade Civil Mamirauá/ Imprensa Oficial do Estado do Pará, 2005. 324 p.

BARNETT, S. et al. Efficacy of pyriprole topical solution against the cat flea, Ctenocephalides felis, on dogs. Veterinary Therapeutics, v. 9, n. 1, p. 4-14, 2008.

CHANDLER, G. T. et al. Fipronil effects on estuarine copepod (Amphiascus tenuiremis) development, fertility and reproduction: a rapid life cycle assay in 96-well microplate format. Environmental Toxicology and Chemistry, v. 23, n. 1, p. 117-124, 2004.

DRYDEN, M. W.; PRESTWOOD, A. K. Successful flea control. Compendium of Continuing Education for the Practicing Veterinarian, v. 15, n. 6, p. 821-831, 1993.

FRANC, M.; YAO, K. P. Comparison of the activity of selamectin, imidacloprid and fipronil for the treatment of cats infested experimentally with Ctenocephalides felis felis and Ctenocephalides felis strongylus. Veterinary Parasitology, v. 143, n. 2, p. 131-133, 2007.

HUTCHINSON, M. J. et al. Evaluation of flea control strategies using fipronil on cats in a controlled simulated home environment. Veterinary Record, v. 142, n. 14, p. 356-357, 1998.

MARCHIONDO, A. A. et al. World Association for the Advancement of Veterinary Parasitology (W.A.A.V.P.) guidelines for evaluating the efficacy of parasiticides for the treatment, prevention and control of flea and tick infestation on dogs and cats. Veterinary Parasitology, v. 145, n. 3-4, p. 332-344, 2007.
MEDLEAU, L. et al. Effect of topical application of fipronil in cats with flea allergic dermatitis. Journal of the American Veterinary Medical Association, v. 221, n. 2, p. 254-257, 2002.

OTRANTO, D.; WALL, R. New strategies for the control of arthropod vectors of diseases in dogs and cats. Medical and Veterinary Entomology, v. 22, n. 4, p.291-302, 2008.

PAYNE, P. A. et al. Effect of 0,29\% w/w fipronil spray on adult flea mortality and egg production of three different cat flea, Ctenocephalides felis (Bouché), strains infesting cats. Veterinary Parasitology, v. 102, n. 4, p. 331-340, 2001.

RUST, M. K. Advances in the control of Ctenocephalides felis (cat flea) on cats and dogs. Trends in Parasitology, v. 21, n. 5, p. 232-236, 2005.

SAMPAIO, I. B. M. Estatística Aplicada à Experimentaçáo Animal. 2 ed. Belo Horizonte: Fundaçáo de Ensino e Pesquisa em Medicina Veterinária e Zootecina, 2002. 265 p.

SCHENKER, R. et al. Comparative speed of kill between nitenpyram, fipronil, imidacloprid, selamectin and cythioate against adult Ctenocephalides felis (Bouché) on cats and dogs. Veterinary Parasitology, v. 112, n. 3, p. 249-254, 2003.

SCHUELE, G. et al. The effect of water and shampooing on the efficacy of a pyriprole $12.5 \%$ topical solution against brown dog tick (Rhipicephalus sanguineus) and cat flea (Ctenocephalidesfelis). Veterinary Parasitology, v. 151, n. 2-4, p. 300-311, 2008.

SCOTT, F. B. et al. Aspectos gerais do controle da pulga Ctenocephalides felis felis em cães. A Hora Veterinária, v. 22, n. 125 , p. 13-18, 2002.

TANNER, P. A. et al. Advances in the treatment of heartworm, fleas and ticks. Canine Practice, v. 22, n. 2-3, p. 40-47, 1997. 